A. Centonza, P. Casagranda, T.J. Owens, J. Cosmas, Y.H. Song, 2007, "Management of Digital Video Broadcasting Services in Open Delivery Platforms”, Int. J. Mobile Communications,

Vol. 5, No. 2, pp186-214

\author{
Published by Inderscience
}

Original Published version:

http://dx.doi.org/10.1504/IJMC.2007.011818

\title{
Management of Digital Video Broadcasting Services in Open Delivery Platforms
}

\begin{abstract}
The future of Digital Video Broadcasting (DVB) is moving towards solutions for efficient delivery of interactive IP multimedia services over DVB networks to handheld terminals. One of the most promising technologies is DVB-Handheld (DVB-H), at present under standardisation. Services deployed via this type of DVB technologies should enjoy reliability comparable to TV services and high quality standards. However, the market at present does not provide effective and economical solutions for the deployment of such services over multi-domain IP networks, due to their high level of unreliability. This paper focuses on service and network management for effective deployment of DVB$H$ services. Experimental results are presented concerning QoS sensitivity to network performance of DVB-H services delivered over a multi-domain IP network. Moreover, a solution for efficient and cost effective service management via QoS monitoring and control and network SLA design is proposed, giving DVB-H operators the possibility of managing service QoS without being tied to third party operators.
\end{abstract}




\section{Introduction}

Digital Video Broadcasting (DVB) technologies are growing quickly, acquiring popularity among end users and opening doors to new multimedia high quality services. So far, such technologies have been mainly used for pure broadcasting of high quality audio/video services (DVB, 2005). DVB services are deployed on dedicated contribution networks with guaranteed performance and reliability, allowing DVB operators to deliver excellent quality of service (QoS) to their end users. However, classical DVB technologies such as DVB-Terrestrial (DVB-T), DVB-Satellite (DVB-S), or High Definition TV (HDTV) (DVB, 2005; Benson and Fink, 1991), do not allow for efficient delivery of services to users on the move and in general are not capable of providing advanced interactive services to such users due to the power consumption characteristics of the terminals and the difficulty of integrating a return channel (Centonza et al., 2006; Cosmas et al., 2004). Indeed, the only successful type of interactive service deployment achieved in the past via DVB technologies was performed on in-car terminals with very high power consumption and therefore limited portability (Heidkamp et al., 2004; Tönjes et al., 2002). Moreover, the fact that classical DVB services are designed to be delivered on dedicated networks using MPEG2 transport stream format (DVB, 2005) constitutes an obstacle to the "all-IP" merging of existing and future technologies in a real convergent framework. This prevents improving the economic efficiency of such platforms and makes it difficult for new content and service providers to be potential actors in the business case scenario. Undoubtedly, by enabling the provisioning of variegate contents and interactive IP services to users on the move, DVB technologies could contribute to the growth of a new market comprising content providers, service providers, network operators, terminal manufacturers and end users, becoming one of the leading wireless technologies for the deployment of high quality services.

For the reasons mentioned above, and with contributions provided by research projects investigating the convergence of cellular mobile and broadcast technologies (Berg et al., 2004; INSTINCT 2005; ENTHRONE, 2005), a new technology has been designed and is currently under standardisation. This technology is called DVB-Handheld (DVB-H) and is aimed at providing an efficient way of carrying interactive IP multimedia services over digital terrestrial broadcasting networks to handheld terminals (Digital Video Broadcasting Handheld, 2004).

One of the issues related with the employment of DVB-H technology is the lack of adequate solutions available on the market for the deployment of interactive rich multimedia services over unreliable 
multi-domain IP networks at reasonably low costs (Jacobs and Davie, 2005; Banitsas et al., 2004). This issue is very important because it affects the achievement of two crucial targets: good economic efficiency of the service delivery platform, and deployment of acceptable QoS, which in turn influences the penetration of the technology. In fact, the most common service level agreements (SLAs) adopted by IP operators at present follow the so-called “insurance approach” (Verma, 2004). In this type of SLA the service provider makes its best attempt to satisfy the performance objectives that are specified in the SLA according to its normal operating procedures. The insurance approach implies that the operator will issue appropriate credits to customers if their SLAs were breached. However, for services like mobile TV deployed on DVB-H terminals, the most important requirement is service continuity, as end users would not tolerate so called "black screens”. Therefore, charging the network operators for under-allocation of service resources would not solve the problem of service interruptions for the end users. This would generate low levels of user satisfaction, as DVB-H services are meant to resemble TV services with respect to their reliability. Moreover, this problem would seriously limit the penetration of DVB-H technology, resulting in high losses of revenue. More recent solutions to SLA management also rely on the penalty charge scheme (Bouillet et al., 2004) and do not guarantee service continuity in the case of a breach of the SLA. New techniques based on the concept of service over networks (SON) and bandwidth brokers (BB) are emerging (Zhenhai Duan et al., 2004; Mantar et al., 2004; Thi Mai Trang Nguyen et al., 2003). Such techniques are very promising but two main drawbacks can be found in the case of DVB-H services. The first drawback is that in order to exploit the advantages of SON and BB solutions a multi-domain contribution network needs to be covered in every IP domain by a SON or BB operator. Although this is not impossible, the authors believe that it will be some time before such a scenario will be achievable and therefore, will not be available at the time of DVB-H commercialisation expected for the middle of 2006. The second drawback is the higher cost of SLAs with SON or BB operators compared to SLAs with network operators. This would harm the economic efficiency of the DVB-H platform and push up the costs of DVB-H services to end-users. A novel and interesting architecture for deployment of IP services over wireless networks has been presented in (Fodil and Pujolle, 2005). This architecture relies on monitoring of QoS and pre-defined policy enforcement at strategic access points along the network. It also gives users the possibility of dynamically configuring the policy criteria to be adopted for their services. However, being "networkoperator centric”, such architecture is not fully adaptable to service management in DVB-H platforms. 
The reason for this is that such a flexible and dynamically configurable network would lead to a higher probability of delays in service resource allocations and resource contention between applications within the same QoS class. Such events would have a high impact on streaming services such as mobile TV, causing interruptions and poor quality of experience. Indeed, service management in DVB$\mathrm{H}$ platforms or more generally, on platforms aimed at delivering multimedia TV-like services, needs to rely on a "DVB platform-centric architecture" where the platform operator does not need to depend on the network provider for QoS management and control. This architecture should give DVB-H operators the opportunity to identify situations of QoS degradation and promptly react in order to guarantee service continuity.

In this paper the problem of DVB-H service management is analysed and novel QoS control solutions and service level agreements (SLAs) for DVB-H networks are proposed. In order to understand the network performance requirements of DVB-H services, the paper presents experimental results concerning the sensitivity of such services to QoS degradation when delivered over multi-domain IP networks. The focus of the paper is on justifying the advantages of the proposed QoS monitoring and control system architecture where the DVB-H operator can directly take an action if the QoS deteriorates beyond acceptable limits and on demonstrating the benefits of novel SLAs proposed for improving the economical efficiency of the platform. The structure suggested for the QoS monitoring and control system allows DVB-H operators to deploy their services using simple and cost effective network SLAs deliberately designed for such purpose, while maintaining high QoS and service continuity. Its novelty is not in the methods used for QoS monitoring or for the choice of measurement points, already adopted in previous works (Fodil and Pujolle, 2005; Kourtis et al., 2004; Habib et al., 2005), but in the deployment of such architecture for service management in DVB systems. Indeed, the way of controlling QoS on DVB-H platforms by acting directly on the service encoding formats, together with the adoption of new SLAs allowing maximisation of the platform-generated revenue constitutes the novelty of the work presented. The remainder of the paper is organised as follows: In Section 2 the effects of multi-domain network performance degradation on DVB-H services are presented. In Section 3 the two main aspects of the designed service management system for DVB-H platforms are presented: the network architecture for QoS monitoring and control and SLA design based on the "reverse penalty charge" technique. Section 4 describes the details of the QoS control 
mechanisms proposed. In section 5 the advantages of the "reverse penalty charge" technique for SLA design are demonstrated. Conclusions are presented in Section 6.

\section{Challenges in DVB-H Services Delivery over Multi-domain Contribution Networks}

In order to understand the problem of QoS degradation for delivery of DVB-H services over multidomain networks, a set of experiments has been carried out. These experiments are aimed at highlighting the need for a dynamic DVB-H platform-centred service management system and at understanding the problems occurring when such dynamic control is not in place. Indeed, the DVB-H standardisation process has suggested a bouquet of services tailored for deployment over DVB-H networks. These services are divided into two main groups: audio/video streaming services, which consist of TV-like services, and data services, which consist of file transfers such as application downloads, electronic service guide download, and Internet services. Two different techniques are used for DVB-H services delivery. In particular, services appertaining to the audio/video streaming group will be delivered using the RTP protocol (Shulzrinne et al., 2003), while data services will be delivered using the FLUTE protocol (Paila et al., 2004). The latter solution is adopted because FLUTE employs forward error correction (FEC) (Carle and Biersak, 2004), which allows for recovery of losses avoiding the use of retransmissions. The different nature of the delivery for these two groups of services results in streaming services having a much higher sensitivity to QoS degradations than data services, as proven by the experimental results described in this section.

The experiments have been carried out aimed at testing service delivery over a multi-domain IPv6 network. The choice of an IPv6 network is motivated by the fact that these experiments should analyse future multi-domain network performance for DVB-H service deliveries and IPv6 is the best candidate for future network protocol formats. The test bed employed consisted of a transmitting server located at Brunel University, London, UK, generating and transmitting the IPv6 packets, and a receiving client, located at the Centre for Research and Technological Innovation of RAI (RAI CRIT), Torino, Italy, receiving and analysing the packets. IPv6 connectivity at the server side was provided via an IPv6 in IPv4 tunnel, while the receiver used a direct IPv6 connection. The tunnel was set up by the Freenet6 Bandwidth Broker and allowed delivery of the traffic along a fixed IPv6 multi-domain route (HEXAGO, 2005). The route was constantly monitored during the experiments and consisted of seven 
hops between sender and receiver. The route characteristics remained unchanged for whole the duration of the tests.

Three sets of experiments were carried out. The first set aimed at loading the multi-domain channel with audio/video streaming-like traces generated using the Iperf application (Iperf, 2005) and studying the channel's performance. The second set of experiments consisted of testing data service delivery on the multi-domain channel using different versions of the FLUTE protocol; the BTFTP protocol (Barz et al., 2004) was also used for comparison of performances in the data service delivery. The third and final set of experiments consisted in audio/video service streaming of multiple MPEG4 traces played at different bit rates. Details of the experimental results are given below.

\subsection{Channel Performance Analysis}

This experiment consisted of using the Iperf application to generate traffic resembling audio/video streams. Several Iperf traces were played simultaneously all of them at 256kbps, while packet loss, delay jitter, and bandwidth statistics were measured at the receiver. It should be noticed that 256Kbps is the encoding bit rate suggested by the DVB-H standardization group for audio/video streaming services. The first result achieved through these tests concerns the maximum bandwidth available on the channel. This bandwidth was between $1.25 \mathrm{Mbps}$ and $1.5 \mathrm{Mbps}$ and was determined by playing single and multiple Iperf UDP traces with an aggregated bandwidth between 1.25Mbps and 1.5Mbps from the sender to the receiver. It was detected that after an interval of time between 30 to 60 seconds the tunnel was closing connection due to a lack of resources available on the channel. This phenomenon was more marked as the traffic aggregate bandwidth increased. The second result obtained concerned QoS monitoring of multiple Iperf traces played simultaneously. Packet loss results are presented here. Indeed, time delay does not have a major impact on DVB-H services QoS due to the time buffering performed at different nodes along the DVB-H chain, while bandwidth availability can be deduced from packet loss statistics in most cases. The results generated with Iperf reveal how QoS degrades proportionally with the aggregate service bandwidth until situations of channel saturation are reached, i.e. overall service bandwidth between 1.25 and 1.5 Mbps. This can be seen from the packet loss percentages shown in Figure 1. The results generated from this experiment can give a clear idea of what would happen to DVB streaming services when the aggregated service bandwidth approaches the allocated bandwidth and no service management mechanisms are in place. 
The packet losses recorded would cause severe degradation of video quality and of user's quality of experience (QoE).

\subsection{Data Service Delivery Analysis}

This set of experiments targeted the performance analysis of unidirectional file transfer protocols for the delivery of DVB-H services over the multi-domain IPv6 channel. Three protocols were tested: FLUTE, Tampere University implementation (Tampere, 2005); FLUTE, INRIA implementation (INRIA, 2005); BTFTP, designed by RAI CRIT and adopted for file transfer over DVB-T networks. The three protocols make use of FEC capabilities. However, they implement different FEC codes (see Table 1).

Tests consisted of six simultaneous transmissions of 26 files of variable length from about $50 \mathrm{kB}$ to about $600 \mathrm{kB}$, each transmission being at a constant bit rate of 256Kbps. After the experiments, the number of files correctly received with the different protocols and FEC codes was recorded; see Table 2. The FEC percentage in Table 2 shows how many FEC packets are inserted every 100 data packets. The BTFTP protocol has a fixed FEC rate and only a 50\% FEC ratio can be used. As can be seen in Table 2, the Low-Density Parity-Check (LDPC) FEC code (INRIA, 2005) is very effective against packet losses. The reason for this is that this type of FEC is designed for the recovery of large losses of consecutive packets. With Reed Solomon FEC code (Carle and Biersak, 2004), the "blocks" of recoverable packets are smaller and it is easier to loose a whole file if a large number of errors occur consecutively. The protocol with the best performance was the INRIA implementation of FLUTE that chooses between Reed Solomon and LDPC based on the file length. Indeed, a further test on the INRIA FLUTE showed that this protocol can match the performance achieved with 50\% FEC redundancy by Tampere Flute just by using 10\% FEC redundancy. BTFTP performs slightly worse than INRIA FLUTE because the "Write Request" packet, including metadata on the file, is not protected by the FEC.

From the results generated it is possible to see how data services and Iperf streaming-like traces have different sensitivities to network performance degradations such as bandwidth restrictions or packet losses. Such differences are due to the fact that the FLUTE protocol, adopted for delivery of data services, uses FEC codes to "protect" the data during transmission, while streaming services are deployed without any protection codes. Therefore, adding a relatively small percentage of redundant information can solve problems caused by network performance degradation and related to data 
services. In the experiment conducted, the minimum FEC percentage to be added in order to recover losses of about $13 \%$ was $20 \%$.

\subsection{Streaming Service Delivery Analysis}

This set of experiments aimed at mirroring the tests illustrated in 2.1 by substituting the Iperf-generated traces with real audio/video streams. The experiments were not meant to give an extensive evaluation of video streaming quality. The objective was to verify and understand the correlation between service QoS degradation and network performance. Four scenarios of multiple audio/video service delivery were run where each audio/video trace was encoded at a bit rate of 256kbps. The scenarios consisted of simultaneous delivery of three, four, five and six audio/video traces on the multi-domain IPv6 network. The audio/video trace adopted was encoded in MPEG4 and contained fast moving sport sequences, slow motion sequences and "talking head" type sequences. The results presented in this section are shown in the form of screenshots of the scenes from the most significant parts of the trace played; see Figure 2. In particular, screenshots from two main sequences are reported, i.e. a basketball match sequence and a "talking head” sequence. Three streaming service scenarios are illustrated in Figure 2. However, it should be noted that the screenshots are purely indicative, giving readers an idea of the whole clip quality, and are not sufficient to give a trustworthy judgement of quality. In Figure 2 it is possible to notice how the quality of the pictures captured degrades as the number of streams played simultaneously increases. Such degradation can be mapped to the network performance recorded during the experiments carried out with Iperf-generated traces, where a very similar type of traffic was deployed along the same route and under the same network conditions. As already mentioned for the results shown in 2.1, the test bed scenario used for delivery of these audio/video traces could be compared to that of a multi-domain contribution network where a shortage of bandwidth is experienced and service resources are under-allocated. This would cause a breach of the SLA contracted between the service operator and the network provider, and therefore a refund issued from the network provider to the service operator. However, such a solution would not eliminate the problem of degraded quality of experience for the end user and of eventual service interruptions.

\section{SLA Design and Service Management}

The results presented in the previous section pointed out that streaming services are more sensitive to network performance degradation than data services, which can fully recover from packet losses thanks 
to the FEC codes adopted. Therefore, the novel DVB-H service management solution proposed in this section mainly applies to streaming services. The solution is made up of two parts: the first part regards the design of special SLAs to be contracted with the different network providers; the second part consists of deploying a system architecture for the implementation of a series of service management actions performed by a QoS monitoring and control system (QoS-MCS) designed to detect and react in real time to those situations in which service QoS degrades beyond acceptable limits, i.e. when a breach of the SLAs contracted with network providers occurs. The core idea behind this system is that of adopting slightly modified and relatively cheap "insurance approach" network SLAs and at the same time enabling DVB-H operators to monitor and actively control their services in order to ensure service continuity.

\subsection{Network SLA Design}

The SLA structure proposed here takes into consideration the overall network performance deriving from concatenation of several network domains involved in the delivery of DVB-H services. SLAs with each network operator will depend on the performance of the overall chain of network domains. Suppose DVB-H streaming services are denoted with $s$ and that the services ingress and egress for a generic domain $d$ are labelled $\sigma_{\mathrm{d}}=\left(\sigma_{\text {ingress }}, \sigma_{\text {egress }}\right)_{\text {Domain d. }}$. Suppose that the aggregate and carried bandwidths for services $s$ and domain $d$ are respectively $\mathrm{U}\left(\mathrm{s}, \sigma_{\mathrm{d}}\right)$ and $\mathrm{V}\left(\mathrm{s}, \sigma_{\mathrm{d}}\right),\left(\mathrm{V}\left(\mathrm{s}, \sigma_{\mathrm{d}}\right) \leq \mathrm{U}\left(\mathrm{s}, \sigma_{\mathrm{d}}\right)\right)$ (Bouillet et al., 2004). It is known that depending on the type of modulation adopted, the maximum overall bit rate available at the radio link for DVB-H transmission on a $5 \mathrm{MHz}$ bandwidth slot is 12Mbps (Digital Video Broadcasting Handheld, 2004). According to the modulation choice of the DVB-H operator and the number and quality of the streaming services, the aggregated bit rate needed for streaming services on one $5 \mathrm{MHz}$ bandwidth slot will assume a certain value, which will be denoted as $R B$. For each network domain $d$ traversed by the aggregated flow of DVB-H streaming services the SLA should state that

$$
\mathrm{V}\left(\mathrm{s}, \sigma_{\mathrm{d}}\right) \geq k^{*} R B, \mathrm{U}\left(\mathrm{s}, \sigma_{\mathrm{d}}\right) \geq k^{*} 12 \mathrm{Mbps}
$$

Where $k$ is an integer denoting how many $5 \mathrm{MHz}$ bandwidth slots the DVB-H operator uses for radio transmission and therefore how many "pipes" of size $R B$ will travel along domain $d$. The ratio $\mathrm{V}\left(\mathrm{s}, \sigma_{\mathrm{d}}\right) / \mathrm{U}\left(\mathrm{s}, \sigma_{\mathrm{d}}\right)$ is the service acceptance ratio for services deployed outside bandwidth $\mathrm{V}\left(\mathrm{s}, \sigma_{\mathrm{d}}\right)$ and within bandwidth $\mathrm{U}\left(\mathrm{s}, \sigma_{\mathrm{d}}\right)$. Each SLA contracted with network domain operators should be designed in such a way that the following condition is respected along the overall chain of delivery: 


$$
\sum_{d} \operatorname{PacketLoss}\left(s, \sigma_{d}\right) \leq \text { MaxPacketLoss }(s)
$$

Where MaxPacketLoss (s) is the maximum packet loss percentage allowed for service $s$.

Due to extensive data buffering performed along a DVB delivery network, packet delays and jitters do not have an influence on service QoS and do not need to be specified in detail within the SLA. Nonetheless, the unacknowledged mode of streaming service delivery in DVB-H networks makes packet loss the most important QoS parameter to be taken into consideration. Packet loss is due to queue overflows in routers or generally routing nodes. However, high percentages of packet loss are usually the consequence of mismatching between the required service bandwidth and the bandwidth offered by the network provider(s).

The service management system proposed for DVB-H platforms is based on the idea that the business model involving DVB-H operators and network providers is such as to allow DVB-H operators to deploy a set of QoS monitoring probes (QoS MPs) at crucial points along the end to end DVB-H service route, i.e. traffic ingress and egress points along each network domain taking part in the service delivery. The choice of the location of monitoring points is based on the structure of QoS monitoring systems presented in (Kourtis et al., 2005) and (Habib et al., 2005). The probes deployed can perform measurements on the service level specifications (SLS) stated in the network SLAs. Whenever the network performance measured by the QoS MPs does not comply with the contracted SLS, i.e. if the carried bandwidth is less then $\mathrm{V}\left(\mathrm{s}, \sigma_{\mathrm{i}}\right)$ and/or the packet loss rate is beyond MaxPacketLoss(s), the probes will monitor this event and report it to a remote unit, where it will be stored together with its duration and location into a database accessible by other nodes in the DVB-H platform. The novelty of network SLAs proposed for delivery of DVB-H services is based on the following:

- The DVB operator will issue a penalty charge to the network operator responsible for the breach of SLA, for any event recorded in the database by the QoS MPs. Such a refund will be called a "reverse penalty charge" and it will increase proportionately with the duration of the breach and/or depending on the "distance” between the SLS agreed in the SLA and the SLS provided by the network operator.

The adoption of a "reverse penalty charge” proposed in this paper constitutes a shift of paradigm with respect to the types of SLA structures used nowadays. Indeed, SLAs used at present rely on the fact that the network provider will monitor the resources allocated to the services traversing its domain and will refund its customers in case of breach of SLA. In the schema proposed, the DVB-H operator is 
able to monitor in real time whether its services are correctly delivered. If this is not the case, an action can be taken in order to fit the services to the resources available on the network, as will be explained in the next section, and a penalty charge can be issued against the network provider breaching the SLA. Hence, service interruptions can be avoided and income loss can be minimised, improving the economic efficiency of the platform.

\subsection{QoS Management System}

The main purpose of the QoS management system is to make service continuity controllable by DVB$\mathrm{H}$ operators, rather than dependent on the network provider(s) performance. By enabling this feature, DVB-H platforms will be "broadcast-operator centric", becoming similar to classical broadcast networks such as DVB-T, where the broadcaster can monitor its services and take measures against unexpected events in real time. A schematic representation of the architecture for the proposed service management system is shown in Figure 3. In Figure 3 QoS MPs are connected to each edge router along the multi-domain service routes, as well as at the ingress (service management system-SMS) and egress (IP encapsulators) of the DVB-H contribution network. QoS MPs will also be located in the broadcasting area in order to receive and analyse services from the broadcast radio channel. QoS MPs have been developed within the INSTINCT project (INSTINCT, 2005) and can perform very accurate audio/video QoS analysis of DVB-H streaming services. The actions taken by the proposed system for QoS monitoring and control are listed in the sequence below:

1) Service Creator (SC) receives the service contents from different sources such as satellite links or raw video streams from a video camera. Such contents are available at different bit rates and encoding formats. According to the choice of the DVB-H operator, such heterogeneous service contents will be transcoded and transrated by the SC into a consistent format and then sent to the SMS for service management and delivery to the IP encapsulators.

2) QoS MPs periodically report QoS statistics to the QoS monitoring and control system (QoSMCS)

3) QoS-MCS stores statistics into a database together with the type of event reported, its location, its time, and the service effected. The QoS-MCS is also aware of the SLS stated in the SLAs contracted by the DVB-H operator with each network provider and of the parameters used for transcoding and transrating of the services at the SC. 
4) QoS-MCS initially uses the database storing all the QoS reports to check if the SC is encoding the services appropriately. If this is not the case, the QoS-MCS will signal the SMS, which in turn will trigger an action on the SC to correct an erroneous service encoding set up.

5) If the services are properly encoded, the QoS-MCS checks whether the measured QoS performance within each IP domain is in accordance with the SLS contracted. If this is not the case, the QoS-MCS will check that the service end-to-end QoS is in accordance with the enduser SLA. If this is the case, the QoS MCS will impose a penalty charge on the network provider(s) that breached the SLS and no further action will be taken.

6) If the service QoS is detected to be below QoS levels stated in the end-user SLA, the QoSMCS signals the SMS to reduce the encoding bit rate of the service effected to a value slightly below the available service bandwidth recorded by the QoS MPs.

7) The SMS will in turn trigger a bit rate reduction action on the service creator and the service bit rate will be reduced.

8) After such a reduction takes place, the SMS will try to slowly increase the service bit rate until the nominal service bit rate is reached. If such small increases result in new channel saturation, the service bit rate will again be reduced below the available bandwidth. This process will hold until the service resources available are in accordance with the SLAs in place and the service bit rate will be set to its nominal value.

9) The SMS will issue a penalty charge proportional to the duration of the breach of the SLAs to each network provider responsible for it.

Communication between QoS MPs and the QoS-MCS has been enabled by designing custom interfaces allowing reporting of Management Information Base (MIB) variables through the Simple Network Management Protocol (SNMP) (Perkins and Mcginnis, 1996). Such interfaces can selectively report the most significant QoS statistics to the QoS-MCS. In the QoS-MCS, the data stored in the database created are grouped according to the time slot they belong to, each time slot being of one minute. All the events captured in each time slot are reported in a XML file, which lists the event titles and their class (i.e. Alarm, Warning, Info), their location (MP address), the time of occurrence, the event state (Start, Continuous, End), the ID number of the service affected (PID), and eventually the details of the measurement (i.e. service bandwidth, delays). If a breach of the end user SLA is detected, the XML file related to the time slot under analysis is sent to the SMS, which will than have all the necessary 
information to trigger an action aimed at controlling the service creation. An example of the XML format used for QoS reporting is shown in Figure 4. In this figure some of the possible events monitored are shown.

\section{On the Fly Service Bit Rate Control}

Experimental results presented in Section 2 revealed how packet losses can generate video quality degradation in DVB-H streaming services. In most of the cases, packet loss rates that constitute a permanent problem for streaming video applications derive from under-allocation of service bandwidth on the multi-domain contribution network. This is due to the fact that the "intelligence" of video players nowadays is such as to enable the recovery of certain percentages of lost video frames without effecting the user's QoE (Albanese et al., 1996; Rhee and Joshi, 2000). Therefore, streaming services can be successfully delivered as long as packet losses remain below the recoverable threshold. However, in cases of service bandwidth denial on the contribution network, streaming services will be effected by a high and continuous packet loss, which will most probably reside outside the recoverable limits. In order to counter react to this type of event, a novel application has been designed and installed in the service management system, aimed at controlling the service encoding bit rate according to the loss rates experienced and service bandwidth available. The application is designed in a way so as not to modify the target service encoding bandwidth if the losses experienced are within recoverable limits and to keep the bandwidth slightly below the bandwidth offered by the network provider(s) during periods of "instability" so as to prevent service interruptions due to bursty oscillations of the bandwidth available. A graphical flow chart representation of the concept behind the “on the fly” bit rate control application is shown in Figure 5.

The application will first be initialized by choosing, the size of the sliding time window over which the service bit rate and the packet loss, the packet loss limit and the target service bandwidth will be averaged. Bandwidth unit and bandwidth rising factors can also be chosen. The application will first check if the average packet loss monitored exceeds the allowed limits. If this is the case, the encoding bit rate will be adjusted to the multiple of the bandwidth unit that is just below the available service bit rate monitored. The parameter "Warning" will then be set to a certain value. This value is the number of time units the application will wait before it starts checking if it is possible to increase again the service bandwidth. The time unit adopted is one second. After expiry of the waiting time, the application will try at every iteration to increase the service bit rate by the bandwidth raising factor. If 
the available bandwidth is lower than the new increased bandwidth and the packet losses exceed the limits, the service bit rate will be reduced accordingly; otherwise it will be increased until the target service bandwidth is reached.

By using the results collected during the experiments described in section 2.1, it has been possible to simulate how the bit rate control application would react to changes in the packet loss rate and service bandwidth monitored on a real multi-domain contribution network. The experiments described in section 2.1 gave the opportunity of collecting packet loss rates and service bandwidth available for each streaming service delivered across the chain of IPv6 domains forming the contribution network. By knowing the behaviour of the packet loss rate and the available service bandwidth and by knowing the initialisation parameters, it is possible to calculate the encoding service bit rate determined by the bit rate control application. Results of the bit rate control simulation can be seen in Figure 6, where the packet loss rate and available service bit rate have been collected via the Iperf application during the experiment described in 2.1 and the initialisation parameters are those specified in Figure 5. In Figure 6-(a) the service bandwidth available on the multi-domain channel is represented in continuous line. If this curve is compared with the packet loss percentage reported in Figure 6-(b), it is possible to see how maximum values of packet losses correspond to minimum values of bandwidth available. As the packet loss threshold has been arbitrarily fixed to $8 \%$, the bit rate control application will not lower the encoding bit rate until packet loss is above such threshold. Therefore, for losses up to $8 \%$ the service encoding bit rate will only be able to increase to or remain constant at the value of the target service bit rate. In Figure 6-(a) the curve in dashed line shows the result of the control performed on the service encoding bit rate. It can be seen how the encoding bit rate stays always below the available bandwidth, except for those cases when packet losses are below recoverable thresholds. In these cases, service bandwidth should not be modified, as the player will be able to recover those losses without effecting the user's QoE. Therefore, the bit rate control application guarantees service continuity at the expenses of a slight degradation of the video quality due to bit rate reduction. However, such video quality degradation does not have a major impact on the user's quality of experience, as will be demonstrated in the following section.

\section{Quality of Experience Analysis}

On-the-fly bit rate control is based on so called bit-drop techniques according to which, as the encoding bit rate is changed, the picture resolution is not modified but the data carried in each video frame is 
reduced, resulting in poorer picture quality. Therefore, if service encoding bit rate control is to be adopted, it is essential to estimate what will be its effect on the end user's quality of experience. For this purpose, a test bed has been set up at Brunel University, resembling the end to end DVB-H delivery platform where the functionalities of the service management system have been implemented for service monitoring and control, as described in the previous sections. The test bed consists of the following components:

- Video Camera: Capturing live video sequences and providing it as raw data.

- Service Creator: An Audio/Video transcoder and transrater receiving the raw traffic generated by the video camera and streaming it in MPEG4 format at a bit rate of 256kbps. The output of the service creator is available as IP traffic.

- Network Emulator (NE): a Linux Debian based network emulator (Debian, 2005), allowing control of packet loss percentages, packet delay, delay jitter and available service bandwidth.DVB-H Gateway: an integrated DVB-H MPEG2 encapsulator and multiplexer providing a MPEG2 transport stream output compliant with the time slicing techniques adopted in DVB-H.

- QoS Monitoring Probe: a MPEG2/IP analyser able to perform extensive analysis of MPEG2 transport streams and IP streams and acting as an agent for reporting the results of such analysis to a remote manager machine via SNMP protocol.

- QoS Monitoring and Control System: a machine performing as SNMP manager collecting SNMP reports from the QoS monitoring probe and issuing alarms in case of anomalous events. In this node information is stored into a database and made available in XML format to the service management system, as explained in Section 3.2.

- DVB-H Terminal: a machine receiving the MPEG2 transport stream coming from the DVB-H gateway and decoding it in accordance with the DVB-H specification. The streaming service was played on such a machine.

A graphical representation of the test bed adopted is shown in Figure 7.

By setting the network performance parameters in the NE, it was possible to reproduce the behaviour of a real multi-domain contribution network such as that analysed in section 2. The experiment performed consisted of two phases: in the first phase the NE is set in such a way as to allow a maximum traffic of $400 \mathrm{Kbps}$ with packet losses following a normal distribution with a mean of $1.5 \%$. Therefore, the streaming audio/video service sent by the SC at 256kbps does not encounter any 
bandwidth restriction and although it is effected by losses it can still guarantee a good quality of picture, see Figure 8-(a). In the second phase of the experiment the NE was set so as to let through traffic limited to 200kbps, while the packet losses were left unchanged. Such event corresponds to an unexpected service bandwidth drop imposed by the network provider(s) in a real network scenario. The effect of such network performance led to service interruption and to the degradation of the picture quality as shown in Figure 8-(b). Under these conditions the QoS MPs started monitoring an “Audio \& Video Bit Rate Lower Than Expected” event, signalling a reduced bit rate of 200kbps. The event information, together with the source address of the probe, the event class, the event status and the service ID were passed to the QoS MCS, which in turn signalled an alarm. As a consequence of this alarm, the encoding service bit rate was reduced to 192Kbps. The effects of such bit rate reduction on the user perceived QoS can be seen in Figure 8-(c). In Figure 8-(c) picture quality deterioration with respect to Figure 8-(a) can be hardly detected. This result should be interpreted in light of the fact that the streaming service analysed consisted of a fast moving audio/video sequence and that such services will be deployed on mobile terminals with a screen size typically of 3.5". Indeed, the quality of the picture when the bit rate is reduced will resemble that of Figure 8-(c) for all the "static" video sequences and for most of the fast moving ones, resulting in a slight degradation for sequences with very fast camera swings. However, such degradation in the picture quality will surely be better accepted by the end users than service interruptions or QoS degradations such as those shown in Figure 8-(b). Therefore, on the fly bit rate control is an effective tool for service management and QoS control in platforms such as DVB-H, where delivery of streaming audio/video services across unreliable contribution networks is performed.

\section{Advantages of SLAs with a 'Reverse Penalty Charge'}

In the previous sections it has been explained how DVB-H platforms should be supported by a service management subsystem and in particular by network SLAs adopting the 'reverse penalty charge', that is to say where the DVB-H operator issues a penalty charge to those network providers that did not comply with the SLS stated in the SLAs. In this section the advantages brought by this type of SLA to the economic efficiency of the platform will be demonstrated. Such improvement in the economic efficiency of the platform could play in favour of end users, whose service tariffs could be lowered making DVB-H services even more attractive. In order to demonstrate such advantages, a scenario is considered where a network operator is charged if the packet loss rate along its network is higher than 
the limit specified in the SLA. This assumption takes into consideration events of service bandwidth denial, as these cases would result in very high packet loss rates. The demonstration is based on the packet loss percentages collected during the experiments illustrated in section 2.1. These percentages refer to the end-to-end performance of the multi-domain contribution network analysed. However, they will be used as if they derived from the network performance of a single network provider. That is the multi-domain network is considered as managed by one network provider contracting one SLA with the DVB-H operator.

\subsection{Income Generated by the 'Reverse Penalty Charge' Scheme}

Assume $\rho_{a v, d}$ is the average aggregated bandwidth needed by DVB-H streaming services travelling along network domain $d$ for the nominal time interval $T$

$$
\rho_{a v, d}=\int_{t}^{t+T} \rho_{d}(t) d t
$$

Where $\rho_{d}(t)$ is the aggregated service bandwidth as a function of time.

Suppose that $\Phi_{d}\left(\rho_{a v, d}\right)$ denotes the cost function for the average aggregated service bandwidth to be purchased by the DVB-H operator from network provider $d$ during the nominal time interval $T$. The time interval $T$ could be one hour, one day, one month etc. Suppose that the maximum allowable packet loss between the ingress and egress of network domain $d$ is $\operatorname{Llim}_{d}$. Suppose that $\pi_{d}(x)$ is the reverse penalty charge per time unit for network domain $d$ as a function of the average loss percentage $x\left(x>\operatorname{Llim}_{d}\right)$ over the time unit. A time unit could be one second. Suppose that $P_{d}(x)$ is the probability that between the ingress and egress of network domain $d$ the percentage of packet loss is $x$, that is $P_{d}(x)$ is the packet loss probability distribution function (PDF) in network domain $d$ :

$$
\operatorname{Pr}(x \geq \text { Loss })=\int_{\text {Loss }}^{100} P_{d}(x) d x
$$

Finally, assume that the average revenue per service $s$ during the nominal time interval $T$ is $R_{s}$.

Given the above assumptions it is possible to determine the utility function of a DVB-H platform delivering streaming services and adopting the reverse penalty charge scheme. Such function a will be called $E_{T}$ and will provide the net income generated by the deployment of streaming services to end users over the nominal time interval $T$.

$$
E_{T}=\sum_{d} R_{d}+\underbrace{\sum_{d}\left[\int_{L L i m_{d}}^{100} \pi_{d}(x) * P_{d}(x) d x\right]}_{W}-\sum_{d} \phi_{d}\left(\rho_{a v, d}\right)
$$


Equation (1) takes into consideration the costs and gains associated with the delivery of streaming services to end users. In this equation the second additive term on the right hand side represents the extra income generated by the reverse penalty charge scheme. This term will be denoted by $W$ and will be analysed in order to understand the influence of the reverse penalty charge on overall net income. The term $W$ depends on the functions $\pi_{d}(x)$, which represent the amount network provider $d$ will have to refund if the average packet loss percentage equals $x\left(x>\operatorname{Llim}_{d}\right)$ over a time unit. It is assumed that function $\pi_{d}(x)$ varies linearly with loss percentage $x$.

$$
\pi_{d}(x)=\left\{\begin{array}{l}
K_{d} * x, x>\text { LLim }_{d} \\
0, \text { otherwise }
\end{array}\right.
$$

The other function contributing to term $W$ is $P_{d}(x)$. In order to determine the PDF that could best fit $P_{d}(x)$, the data collected during the experiments described in section 2.1 have been used. The data were collected during the simultaneous delivery of six Iperf-generated traces, each of them played at 256kbps, and consist of packet loss percentage values each obtained as the average packet loss experienced by a trace over one second. The PDF that was found to best fit the raw data is a Normal distribution with mean $\mu=10.0758$ and standard deviation $\sigma=7.36411$. Assuming that all network domains are affected by the same packet loss characteristics, the normal distribution obtained will be chosen to represent the function $P_{d}(x)$. A plot of the cumulative distribution function for the raw data and for the Normal distribution selected is shown in Figure 9, where the accuracy of the Normal distribution approximating the data can be appreciated.

By substituting the PDF fitted to the raw data to function $P_{d}(x)$ it is possible to derive term $W$. In order to do so the following equivalences will be used:

$$
\begin{gathered}
\beta=\frac{K_{d}}{\sigma \sqrt{2 \pi}} ; \\
\alpha=-\frac{1}{2 \sigma^{2}} ; \\
\frac{1}{\sigma \sqrt{2 \pi}} \int_{L L i m_{d}}^{100} e^{-\frac{(x-\mu)^{2}}{2 \sigma^{2}}} d x=\frac{1}{2}\left[1+\left.\operatorname{erf}\left(\frac{x-\mu}{\sigma \sqrt{2}}\right)\right|_{L L i m_{d}} ^{100}\right]
\end{gathered}
$$

Term $W$ can be derived as follows: 


$$
\begin{aligned}
& W=\sum_{d}\left[\int_{\text {LLim }_{d}}^{100} K_{d} * x^{*} \frac{1}{\sigma \sqrt{2 \pi}} e^{-\frac{(x-\mu)^{2}}{2 \sigma^{2}}} d x\right]= \\
& =\sum_{d}\left[\frac{\beta}{2 \alpha} \int_{L L i m_{d}}^{100} 2 \alpha^{*}(x-\mu)^{*} e^{\alpha(x-\mu)^{2}} d x+\frac{\beta}{2 \alpha} \int_{\text {LLim }_{d}}^{100} \mu^{*} e^{\alpha(x-\mu)^{2}} d x\right]= \\
& =\sum_{d}\left[-\left.\frac{K_{d} \sigma}{\sqrt{2 \pi}} * e^{-\frac{(x-\mu)^{2}}{2 \sigma^{2}}}\right|_{\text {LLim }_{d}} ^{100}+\left.\frac{K_{d} \mu}{2} * \operatorname{erf}\left(\frac{x-\mu}{\sigma \sqrt{2}}\right)\right|_{\text {LLim }_{d}} ^{100}\right]
\end{aligned}
$$

Where the erf function is defined as

$$
\operatorname{erf}(x)=\frac{2}{\sqrt{\pi}} \int_{0}^{x} e^{-x^{2}} d x ;
$$

Term $W$ can now be analysed in order to determine its influence on the overall income generated by delivery of streaming services on the DVB-H platform. To do so, it is assumed that the contribution network is made of one network domain only $(d=1)$ and that the constant $K_{d}$ is fixed to one, which means that the reverse penalty charge for domain $d$ increases linearly with the loss percentage and with a slope equal to one. It should be highlighted that the choice of how reverse penalty charges vary with loss percentages is up to the DVB-H operator, or any operator managing the service delivery platform. Such charges could follow a trend with different slopes or even a trend with non-linear characteristics, i.e. exponential. The expression derived in equation (2) for term $W$ allows it to be plotted against loss percentages and for different values of $\mu$ and $\sigma$. These graphs are shown in Figure 10 and have been obtained by setting the loss limit $\operatorname{Llim}_{d}$ to $8 \%$.

The results obtained from the analysis of the term $W$ show how the income generated by the reverse penalty charge increases if the mean $\mu$ of the packet loss PDF increases (Figure 10-(a)). However, as an increase in $\mu$ would result in poorer QoS, a trade off has to be made within the SLAs contracted to fix a maximum allowed value of $\mu$. Such a trade off should be made to maximise the overall income generated. In Figure 10-(b) it is shown how the function represented by term $W$ varies as the standard deviation of the packet losses increases. It can be noticed that the revenue generated by the reverse penalty charge decreases if the standard deviation of the loss percentages increases. This increase would cause a drop in overall net income as well as complications in the service management due to very high bursts of packet losses, which are difficult to compensate for in real time. Therefore, in the case of adoption of the reverse penalty charge, the SLAs contracted with network providers should be designed in such a way as to fix low values for the packet loss standard deviation allowed, which 
would not harm the economic efficiency of the platform and would still guarantee QoS control in cases of unexpected network performance degradation.

\section{Conclusions}

In this paper a novel solution for service management in platforms delivering TV-like services over unreliable contribution networks is presented. The services taken into consideration are streaming services, as they are more vulnerable to network performance degradation than data services. The main two aspects of the service management system proposed are a network architecture allowing the platform operator to monitor its services remotely and to be able to trigger control actions in case of unexpected network behaviour and customised SLAs designed in such a way as to improve the economic efficiency of the platform and maintain good QoS at the end user terminal. The idea behind the service management system proposed is that of a "service-operator centric" delivery platform, where service continuity and QoS do not have to depend on the performance provided by network providers involved in the service distribution. The paper has presented experimental results showing what could happen to sensitive services such as streaming audio/video if a service management system was not in place. Also, results were presented demonstrating how the system proposed can guarantee good quality of experience for the end users and how it can improve the economic efficiency of the delivery platforms. Future work will focus on integrating the proposed service management system on hybrid broadcast-cellular networks, in order to provide monitoring and control of services delivered on both broadcast and cellular telecommunications channels to the same end user terminal.

\section{Acknowledgment}

The partial funding of this work by the European Commission through the IST Integrated project INSTINCT contract number 507014 is gratefully acknowledged.

\section{References}

DVB (2005) The Standard of the Digital World, Last Access 23 May, www.dvb.org

Benson, K.; Fink, D.,(1991) 'HDTV’, Multiscience Press

Centonza, A.; Taylor, G.A.; Itagaki, T.; Owens, T.J.; Cosmas, J.; Song, Y-H. (2006) 'Stochastic user behaviour modelling and network simulation for resource management in cooperation with mobile telecommunications and broadcast networks', International Journal of Mobile Communications, forthcoming. 
Cosmas, J., Itagaki, T., Krishnapillai, K., Lucas, A. (2004) 'Multimedia broadcast and internet satellite system design and user trial results’, International Journal of Services and Standards, Vol.1, No 3, pp. 336 - 357.

Heidkamp, B.; Pohl, A.; Schiek, U.; Klinkenberg, F.; Hynynen, J.; Sieber, A.; Christ, P.; Owens, T.J.; Cosmas, J.; Itagaki, T.; Sun, F. (2004) 'Demonstrating the feasibility of standardised application programme interfaces that will allow mobile/portable terminals to receive services combining UMTS and DVB-T', International Journal on Services and Standards, Vol.1, No 2, pp.228 - 242.

Tönjes R. Moessner, K., Lohmar, T., Wolf, M., (2002) 'OverDRiVE - Spectrum Efficient Multicast Services to Vehicles’, IST Mobile Summit, Thessaloniki, 16-19.June.

Berg, M., Butterfield, S., Cosmas, J., Casagranda, P., Garrec, D., Guiraudou, M., Martinez, G., Launay, E., Mazieres, B., Milanesio, D. (2002) 'CISMUNDUS: Convergence of Digital Broadcast \& Mobile Telecommunications', IBC2002 Conference, Amsterdam, September.

Digital Video Broadcasting Handheld (2004) ‘Transmission System for Handheld Terminals (DVB-H)’, ETSI EN 302304 V1.1.1.

INSTINCT (2005) INSTINCT for Convergence, Last Access 23 May, www.ist-instinct.org

ENTHRONE (2005) End-to-End QoS through Integrated Management of Content, Networks and Terminals, Last Access 23 May, www.enthrone.org

Jacobs, P.; Davie, B. (2005) 'Technical Challenges in the Delivery of Interprovider QoS', Communication Magazine, IEEE, Vol. 43, Iss. 6, pp. 112 - 118.

Banitsas, K.A., Song, Y-H, Owens T. J. (2004) 'OFDM over IEEE 802.11b hardware for telemedical applications', International Journal on Mobile Communications, Vol.2, No 3, pp. 310 - 327.

Verma, D.C. (2004) 'Service level agreements on IP networks', Proceedings of the IEEE, Vol. 92, Iss. 9, September, pp. 1382 - 1388

Bouillet, E.; Mitra, D.; Ramakrishnan, K.G., (2004) 'The structure and management of service level agreements in networks', Journal on Selected Areas in Communications, IEEE, Vol 20, Iss. 4, pp. 691 - 699

Zhenhai Duan; Zhi-Li Zhang; Hou, Y.T. (2003) 'Service overlay networks: SLAs, QoS, and bandwidth provisioning’, Transactions on Networking, IEEE/ACM, Vol. 11, Iss. 6, pp. 870 - 883

Mantar, H.A.; Junseok Hwang; Okumus, I.T., Chapin, S.J. (2004) 'A scalable model for interbandwidth broker resource reservation and provisioning', Journal on Selected Areas in Communications, IEEE, Vol 22, Iss. 10, pp. $2019-2034$.

Thi Mai Trang Nguyen; Boukhatem, N.; Pujolle, G. (2003) 'COPS-SLS usage for dynamic policy-based QoS management over heterogeneous IP networks', Network, IEEE, Vol. 17, Iss. 3, pp. 44 - 50.

Fodil, I.; Pujolle, G. (2005) 'Roaming and service management in public wireless networks using an innovative policy management architecture’, International Journal of Network Management, Vol. 15, Iss. 2, pp. 103 - 121. 
Kourtis, A., Skianis, C., Kormentzas, G., Xilouris, G., Negru, D., Mehaoua, A., Ahmed, T., Borcoci, E., Asgari, H., Eccles, S., LeDoeuff, E. (2004) 'Provisioning of End to End QoS in Diverse Environments: The ENTHRONE View’, WSEAS Transactions on Communications, Vol. 3, Iss. 2, ISSN 1109-2742, pp. 626-631.

Habib, A.; Fahmy, S; Bhargava, B. (2005) 'Monitoring and controlling QoS network domains', International Journal of Network Management, Vol. 15, Iss. 1, pp. 11 - 29.

Schulzrinne, H.; Casner, S.; Frederick, R.; Jacobson, V. (2003) 'RTP: A Transport Protocol for Real-Time Applications', RFC 3550.

Paila, T.; Luby, M.; Lehtonen, R.; Roca, V.; Walsh, R. (2004) 'FLUTE - File Delivery over Unidirectional Transport', RFC 3926.

Carle, G.; Biersack, E.W. (1997) 'Survey of error recovery techniques for IP-based audio-visual multicast applications', Networks, IEEE, Vol. 11, Iss. 6, pp. 24-36.

HEXAGO (2005) IPv6 Deployment Today, Last Access 23 May, www.hexago.com.

Iperf (2005) NLANR Distributed Applications Support Team, Last Access 23 May, http://dast.nlanr.net/Projects/Iperf/

Barz, C.; et al. (2004) 'Deliverable 14 - Description of Demonstrator for Mobile Multicast and the Vehicular Router', Annex C, IST OverDRiVE Project.

Tampere (2005) MAD Project’s Home Page, Last Access 23 May, http://atm.tut.fi/mad/

INRIA (2005) MCLv3: an Open Source Implementation of the ALC and NORM Reliable Multicast Protocol, Last Access 23 May, http://www.inrialpes.fr/planete/people/roca/mcl/mcl.html

Perkins, D; Mcginnis, E. (1996) Understanding SNMP-MIB, Prentice Hall Publisher.

Albanese, A.; Blomer, J.; Edmonds, J.; Luby, M.; and Sudan, M. (1996) 'Priority encoding transmission', Transactions on Information Theory, IEEE, Vol. 42, Iss. 6, Part 1, pp. 1737 - 1744.

Rhee, I.; Joshi, S.R. (2000) 'Error recovery for interactive video transmission over the Internet', Journal on Selected Areas in Communications, IEEE, Vol. 18, Iss. 6, pp.1033 - 1049.

Debian (2005) Debian Home Page, Last Access 23 May, http://www.debian.org 


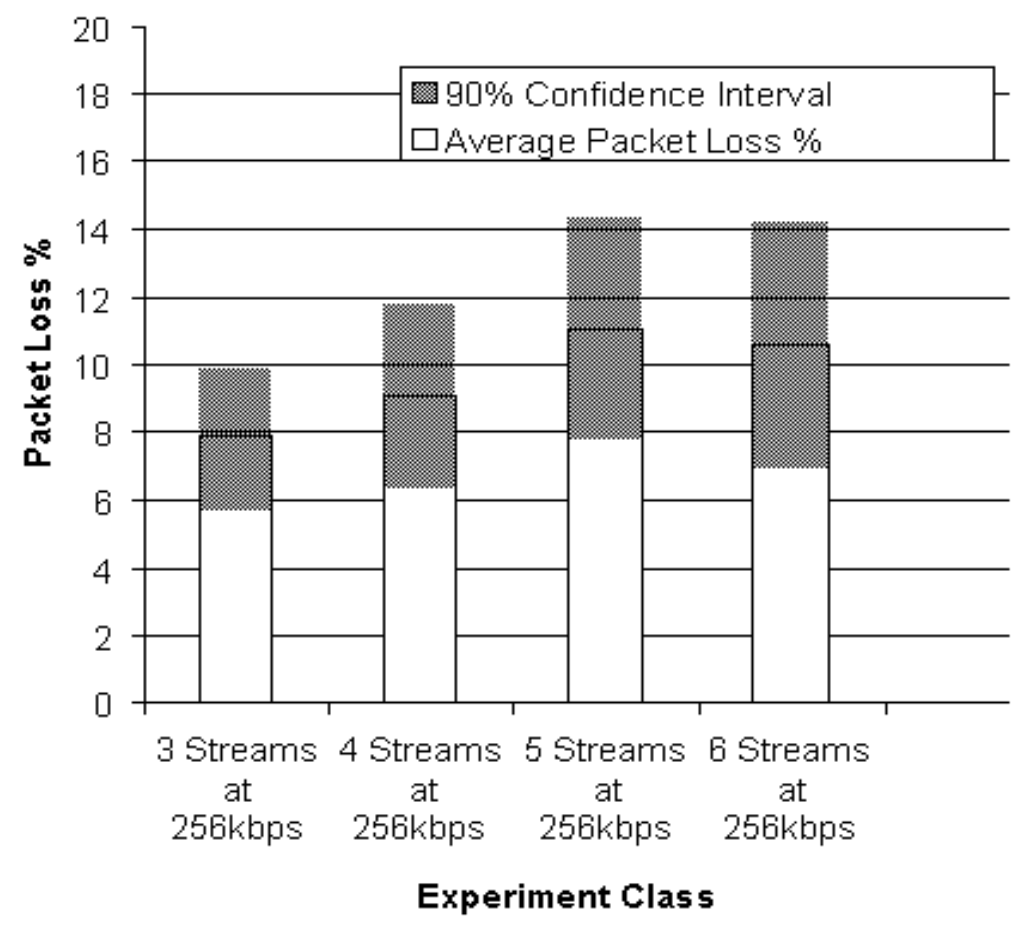

Figure 1: Test results using the Iperf application 


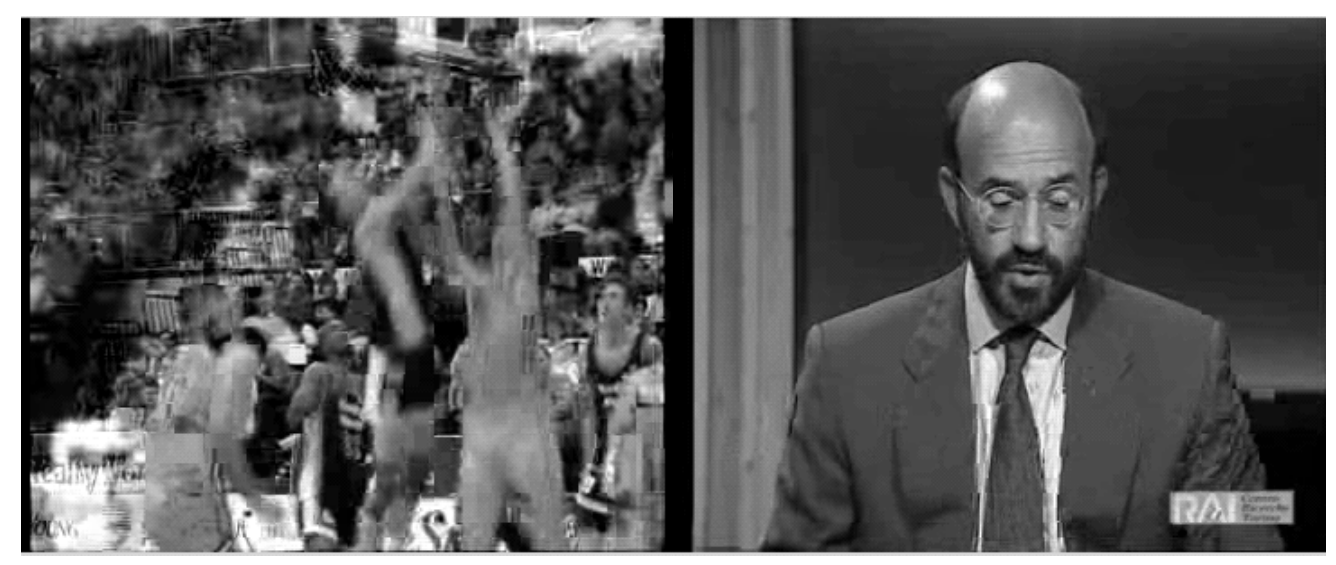

2(a)

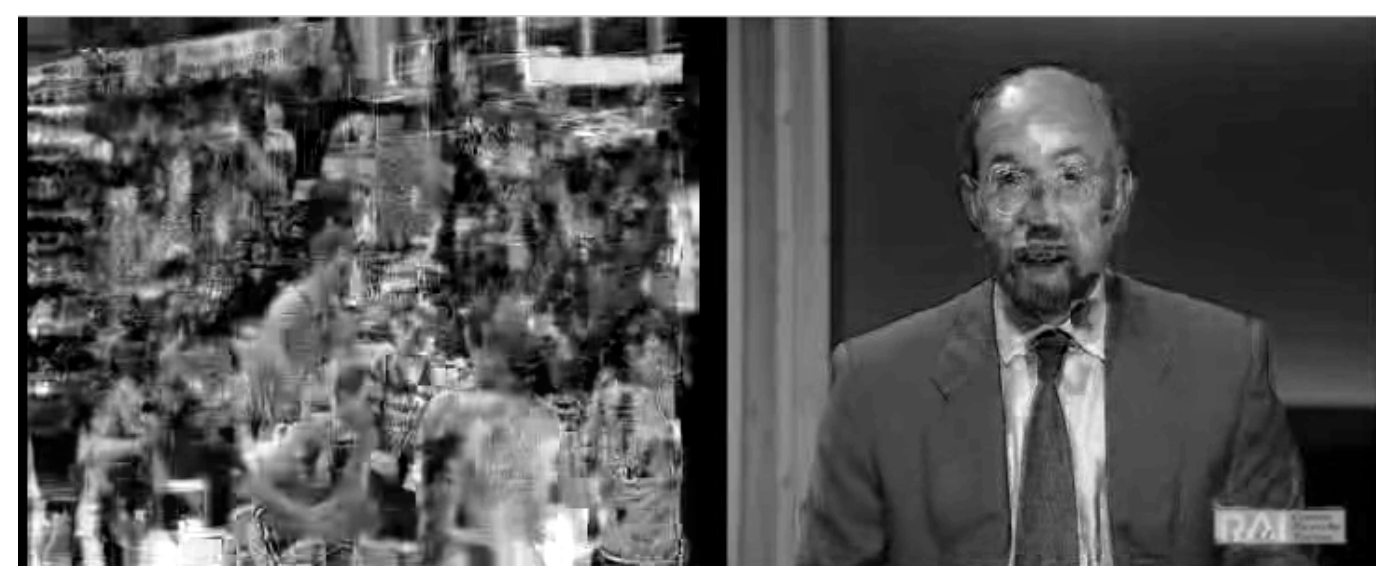

2(b)

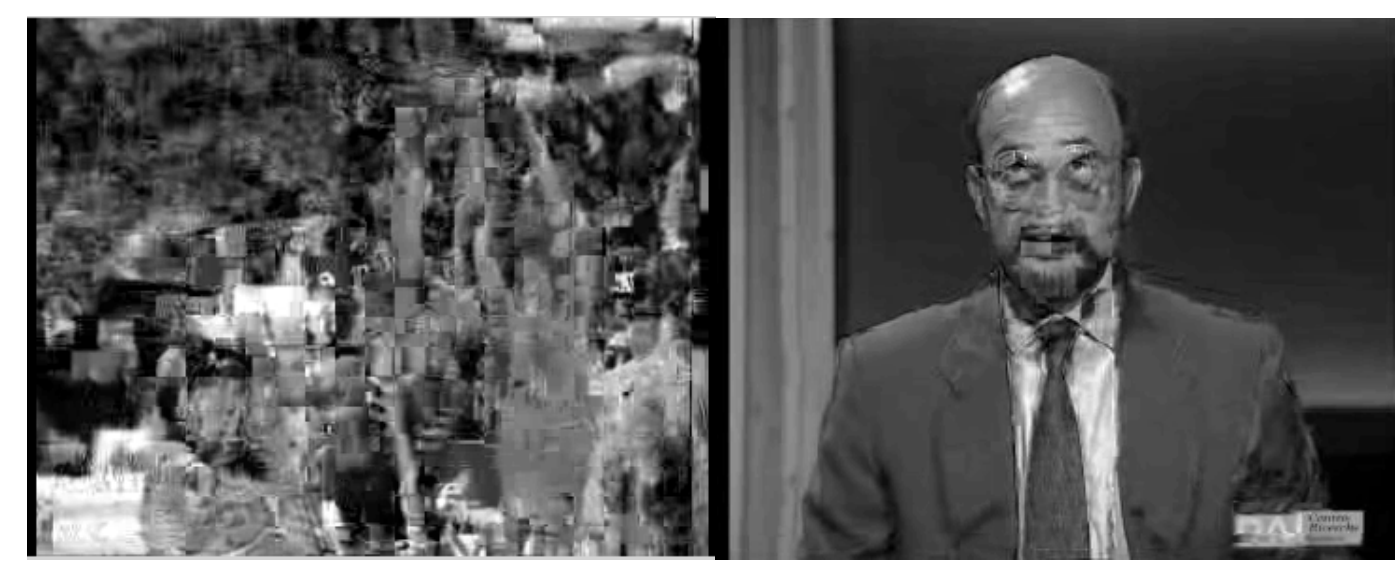

2(c)

Figure 2: Screenshots of a fast moving sequence (left) and of a "talking head" sequence (right) under different network load conditions. 2-(a): 3 streams played simultaneously at 256kbps. 2-(b): 5 streams played simultaneously at 256kbps. 2-(c): 6streams played simultaneously at 256kbps 


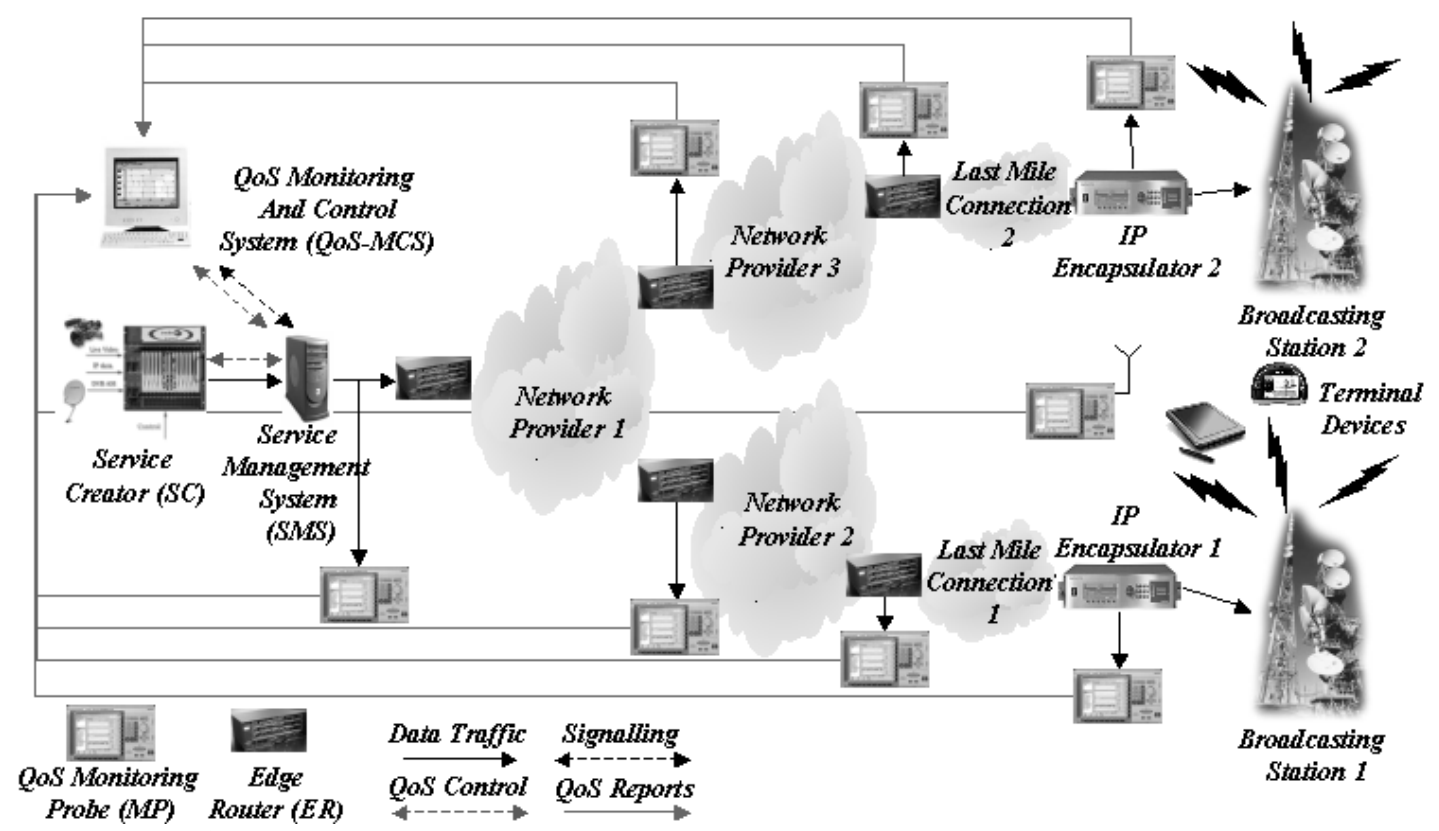

Figure 3: Network architecture of the proposed service management system

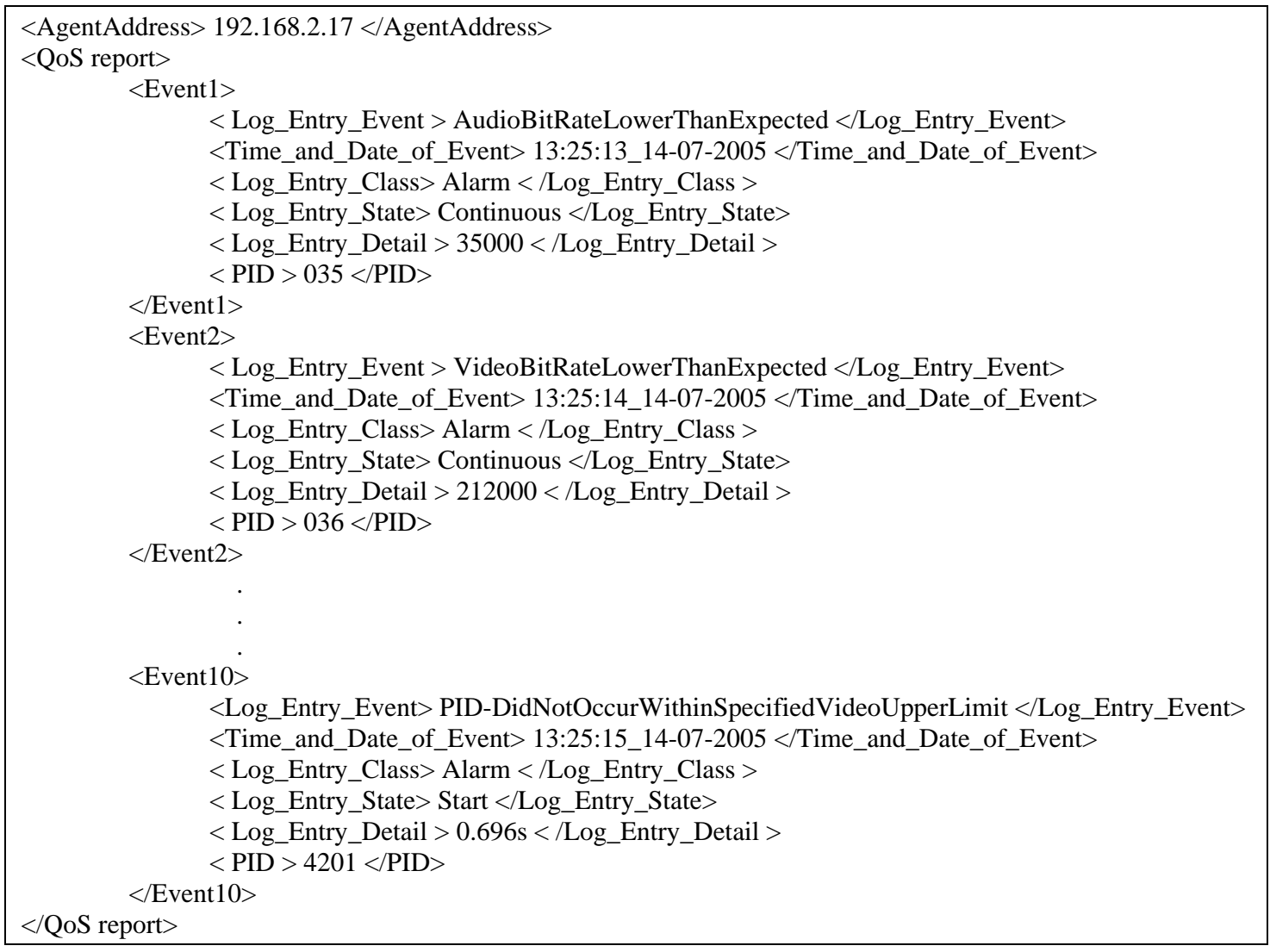

Figure 4: Example of an XML report for the first ten events occurring in a one-minute time slot 


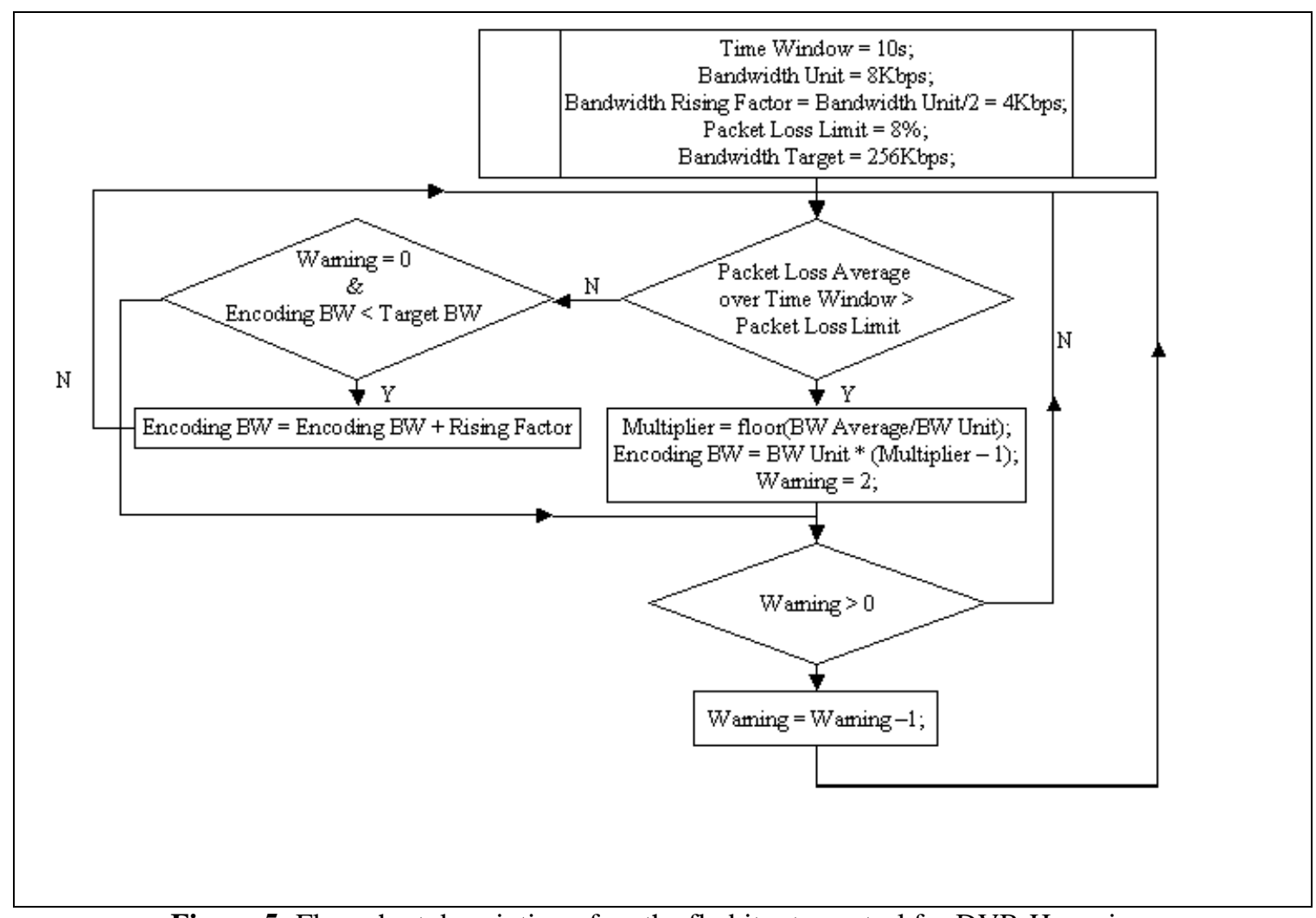

Figure 5: Flow chart description of on the fly bit rate control for DVB-H services 

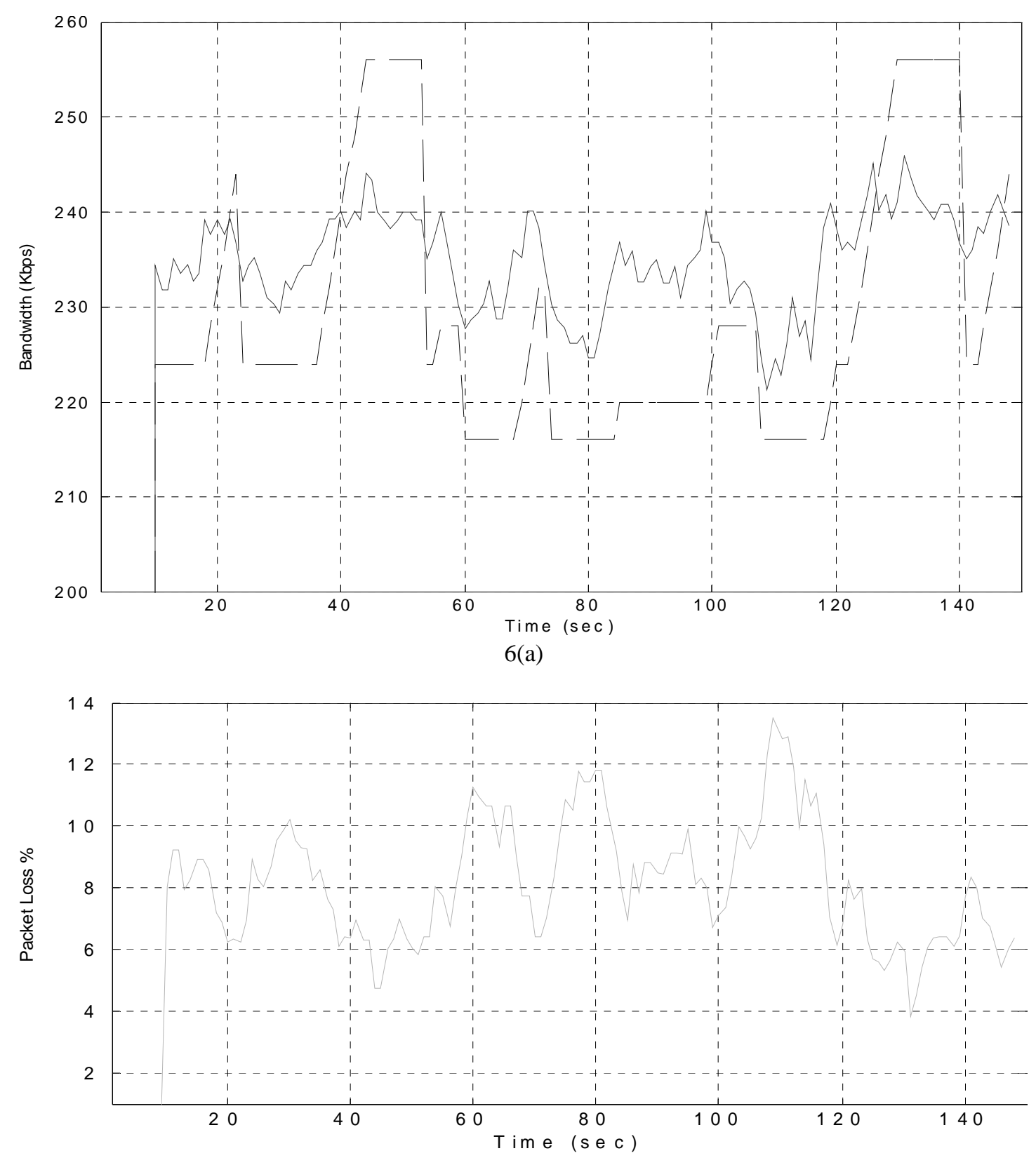

6(b)

Figure 6: Simulation of "on the fly" bit rate control. The curve in continuous line of Figure 6-(a) represents the available service bandwidth, while the curve in dashed line represents the service encoding bit rate as modified by the bit rate control application. In Figure 6-(b) the packet loss percentage is shown. 


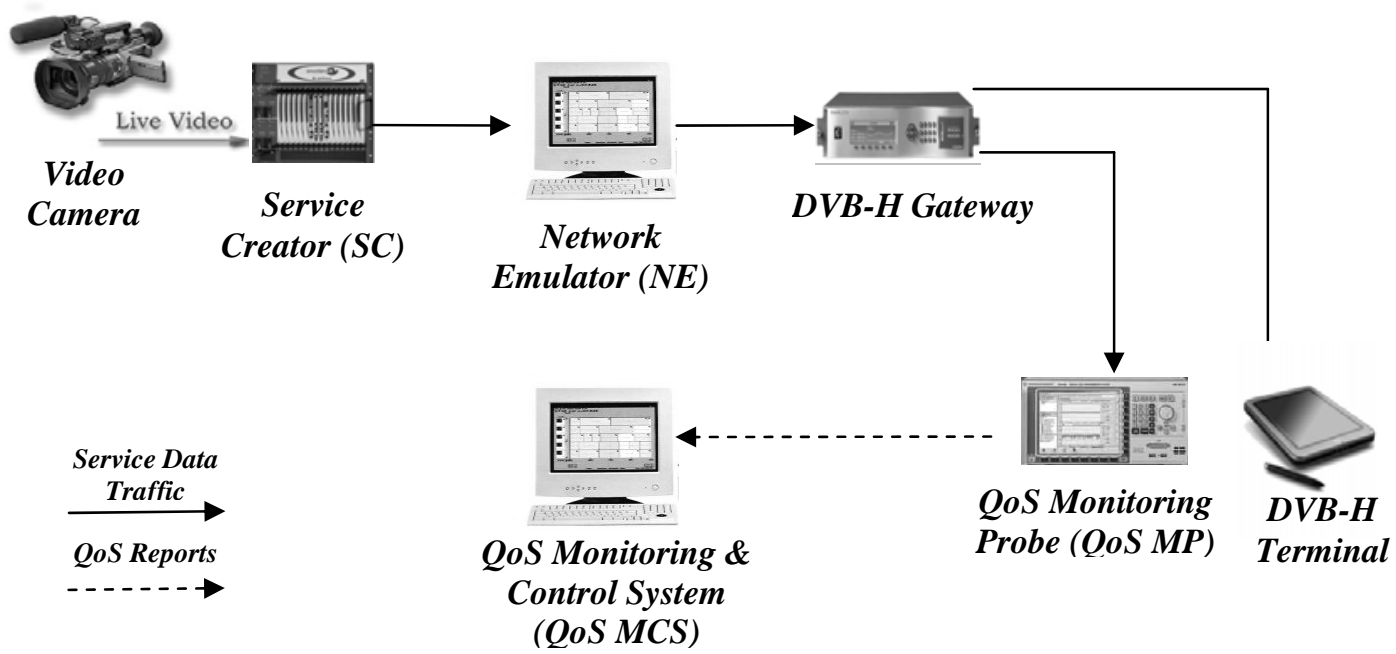

Figure 7: Test bed scenario adopted for experiments on "on the fly” bit rate control

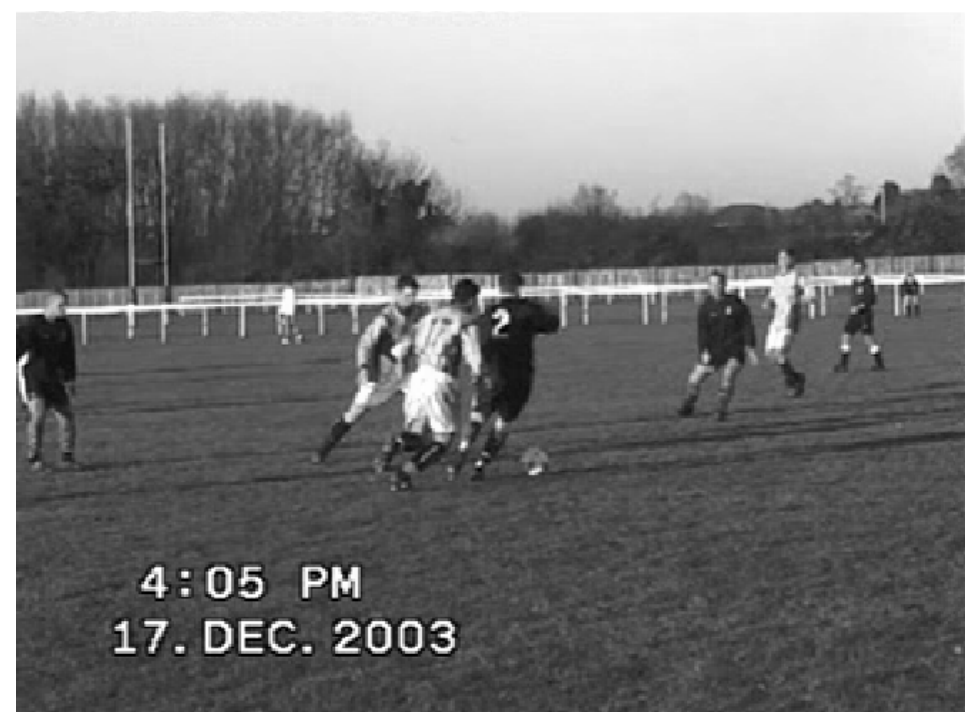

8(a) 


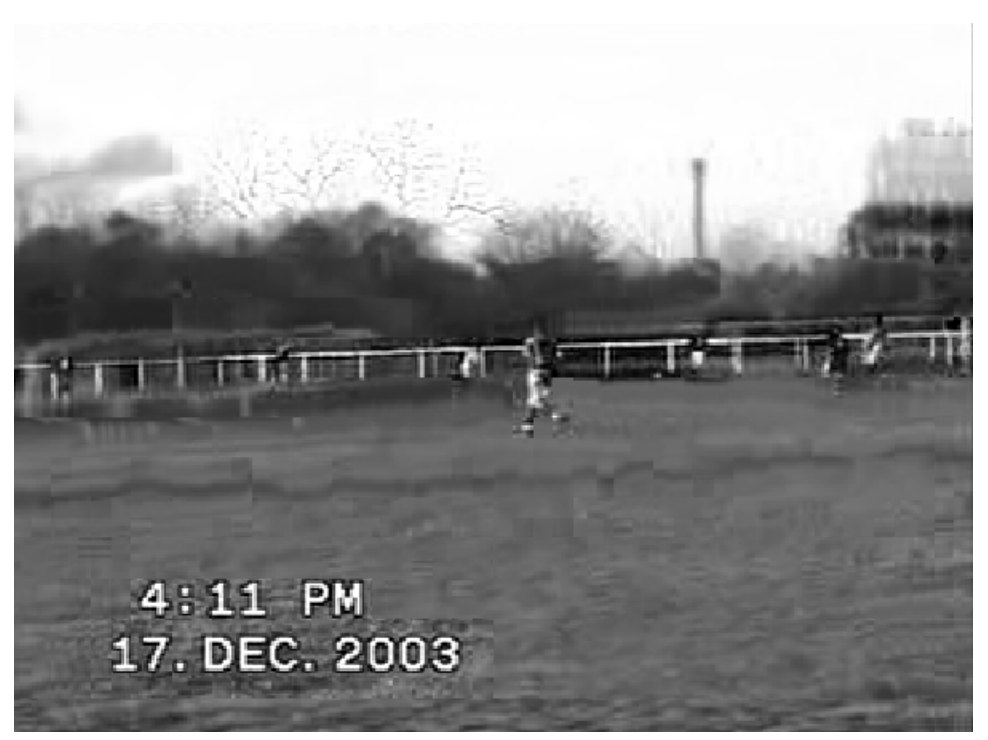

8(b)

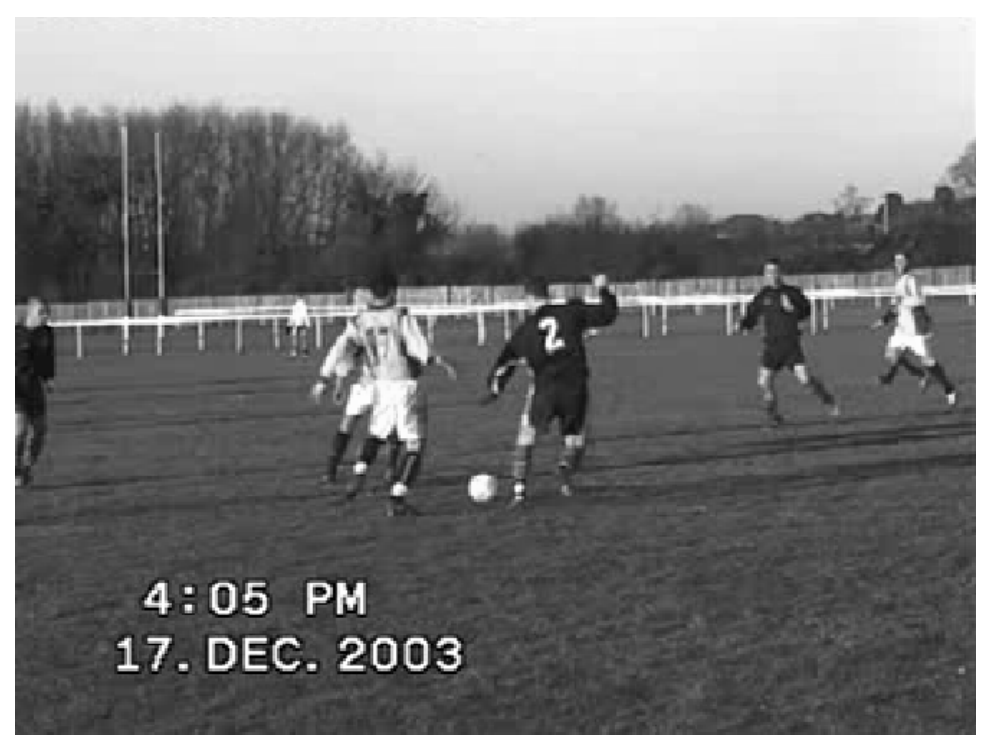

8(c)

Figure 8. Picture quality analysis when on the fly bit rate control is adopted. 8-(a) Video stream encoded at 256kbps, no bandwidth restrictions are emulated; 8-(b) video stream encoded at 256kbps, service bandwidth available is $200 \mathrm{kbps}$; 8-(c) video stream encoded at 192kbps, service bandwidth available is $200 \mathrm{kbps}$ 


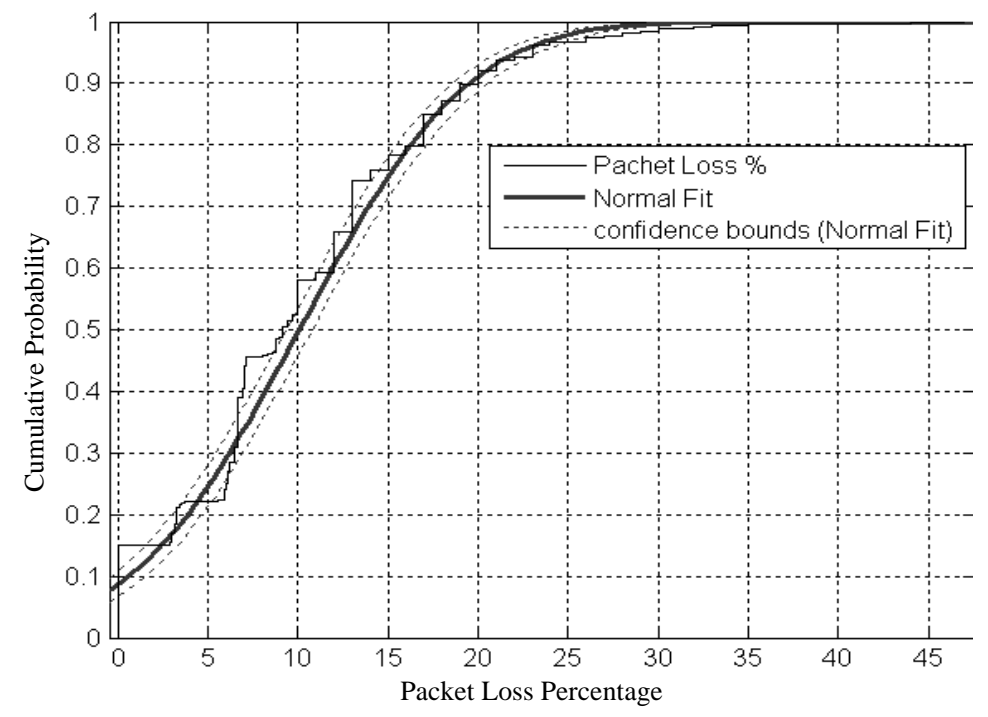

Figure 9: Cumulative distribution of the raw data analysed (single line) and the fitted distribution function (double line). The dotted lines represent the $99 \%$ confidence bounds for the fitted distribution function.

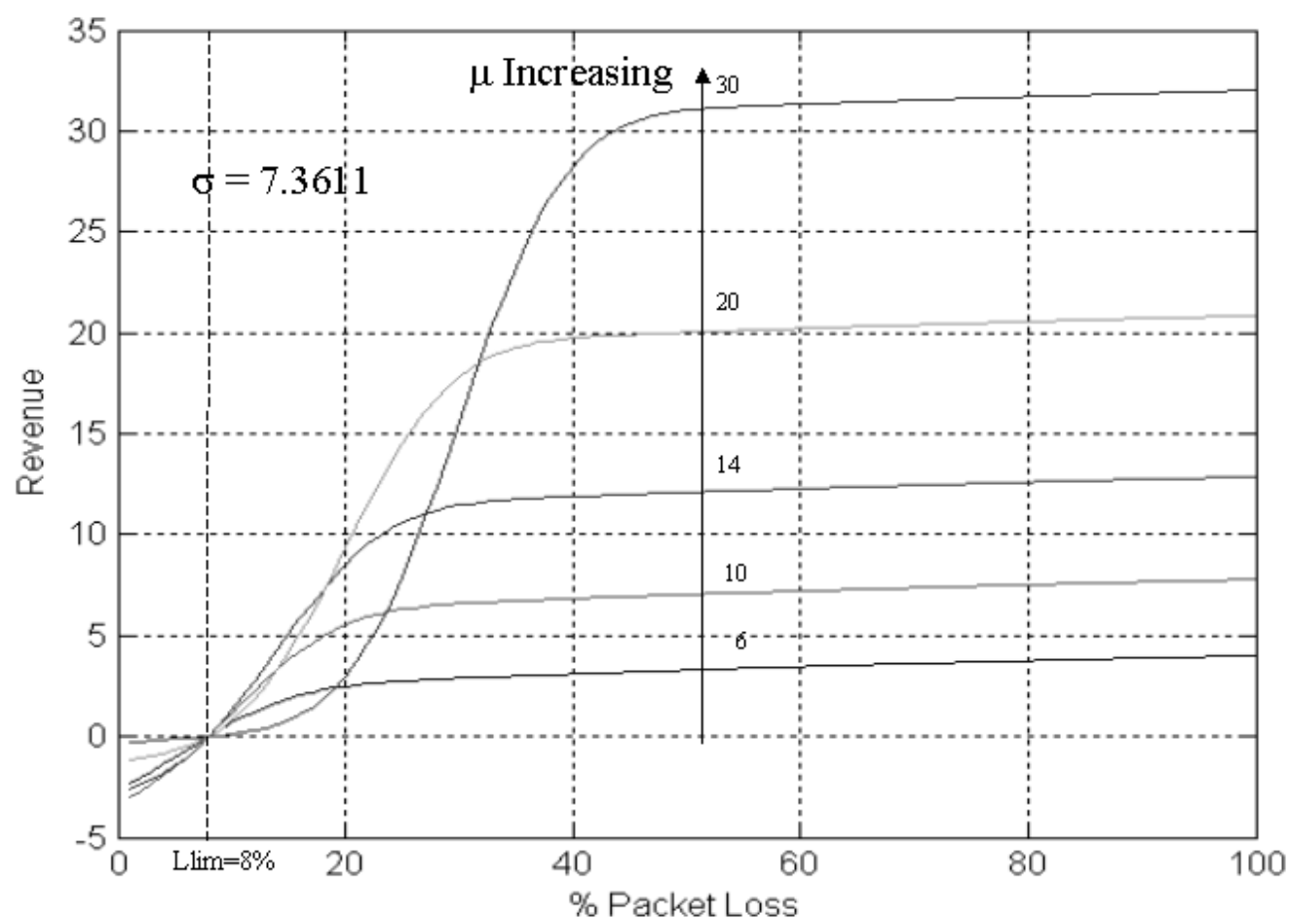

10(a) 


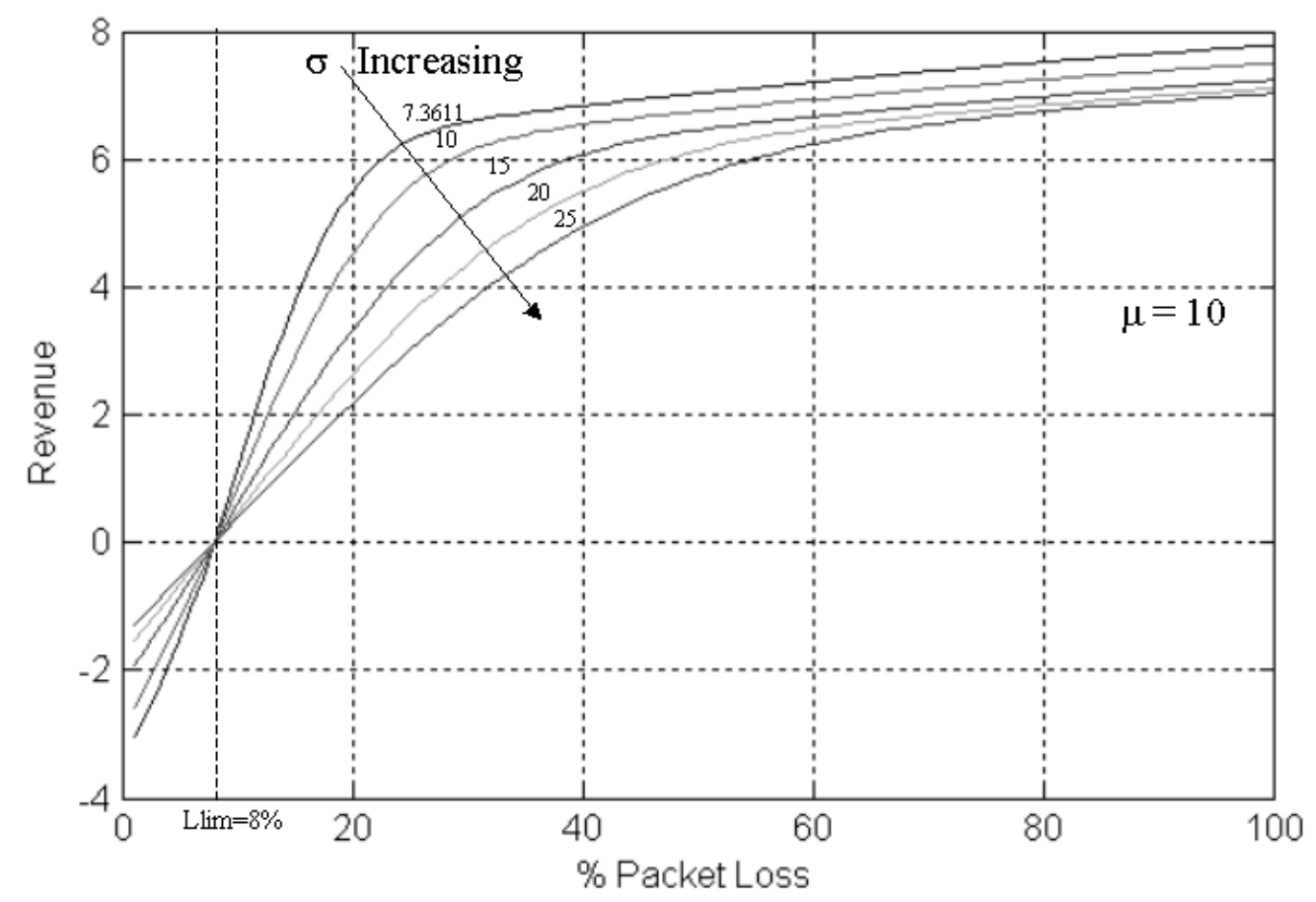

10(b)

Figure 10. Net income generated by the reverse penalty charge technique. 10-(a) Influence of the average packet loss $\mu$ on the net income. 10-(b) Influence of the packet loss standard deviation $\sigma$ on the net income. 
Table 1: FEC implementations in tested file transfer protocols

\begin{tabular}{|c|c|c|}
\hline Protocol Implemented & FEC Scheme & FEC Packet Length \\
\hline Tampere University - FLUTE & Reed Solomon & 1 Kbyte \\
\hline INRIA - FLUTE & $\begin{array}{c}\text { Reed Solomon for small files, } \\
\text { LDPC for big files }\end{array}$ & 1 Kbyte \\
\hline $\begin{array}{c}\text { RAI CRIT - BTFTP (Broadcast } \\
\text { Trivial FTP) }\end{array}$ & LDPC & 0,8 Kbyte \\
\hline
\end{tabular}

Table 2: Test results for six simultaneous 256kbps data service deliveries with file transfer protocols implementing different FEC codes

\begin{tabular}{|c|c|c|c|c|c|}
\hline \multicolumn{2}{|l|}{ Tampere FLUTE } & \multicolumn{2}{l|}{ INRIA FLUTE } & \multicolumn{2}{l|}{ BTFTP } \\
\hline $\begin{array}{c}\text { FEC } \\
\text { Ratio }\end{array}$ & $\begin{array}{c}\text { Received } \\
\text { Files } \%\end{array}$ & $\begin{array}{c}\text { FEC } \\
\text { Ratio }\end{array}$ & $\begin{array}{c}\text { Received } \\
\text { Files \% }\end{array}$ & $\begin{array}{c}\text { FEC } \\
\text { Ratio }\end{array}$ & $\begin{array}{c}\text { Received } \\
\text { Files \% }\end{array}$ \\
\hline $\begin{array}{c}\text { No } \\
\text { FEC }\end{array}$ & $2 \%$ & No FEC & $2 \%$ & $\begin{array}{c}\text { No } \\
\text { FEC }\end{array}$ & $0 \%$ \\
\hline $20 \%$ & $26.3 \%$ & $20 \%$ & $100 \%$ & N/A & N/A \\
\hline $34 \%$ & $73.1 \%$ & $34 \%$ & $100 \%$ & N/A & N/A \\
\hline $50 \%$ & $94.2 \%$ & $50 \%$ & $100 \%$ & $50 \%$ & $100 \%$ \\
\hline
\end{tabular}

